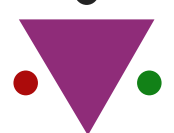

IJCRR

Section: Healthcare Sci. Journal Impact Factor: 6.1 (2018) ICV: 90.90 (2018)

(c) (i) (3)

Copyright@IJCRR

\section{Perception and Awareness of COVID-19 Among Urban and Rural Population - A Survey}

\section{J. Dhayanithi' ${ }^{1}$, Balaji Ganesh S2 ${ }^{2}$ M. P. Brundha ${ }^{3}$}

'Saveetha Dental College and Hospitals, Saveetha Institute of Medical and Technical Sciences, Saveetha University, Chennai - 77, Tamilnadu, India; ${ }^{2}$ Scientist, White Lab - Materials Research Centre, Saveetha Dental College and Hospitals, Saveetha Institute of Medical and Technical Sciences, Saveetha University, Chennai - 77, Tamilnadu, India; ${ }^{3}$ Associate Professor, Department of General Pathology, Saveetha Dental College and Hospitals, Saveetha Institute of Medical and Technical Sciences, Saveetha University, Chennai-77, Tamilnadu, India.

\title{
ABSTRACT
}

Introduction: SARS-CoV-2 is one of seven types of coronavirus. Infections caused by them range from mild to deadly. Some complications of COVID-19 can be caused by a condition known as cytokine release syndrome or cytokine storm. The patients with COVID-19 should be protected from nosocomial infection in hospitals. This study mainly aims to know about the awareness and understanding of rural and urban populations about the COVID-19 virus.

Materials and Methods: A total of 100 participants were involved in this survey. Participants in the study belong to rural (Ammapettai, Kachirapalayam - Kallakurichi district of Tamil Nadu) and urban (Trichy, Salem, Madurai, and Chennai cities) places of Tamil Nadu, India. The questions were simple and understandable and it was distributed through WhatsApp and the Google forms. Data was collected from filled questionnaires and analyzed using SPSS software.

Results: From the 100 responses, $53 \%$ of the study population was urban and $47 \%$ belong to rural areas. $66 \%$ of participants accept that urban populations are more prone to the spread of coronavirus infection than the urban population. $60 \%$ of participants agree that both urban and rural people are aware of coronavirus. $65 \%$ of participants say that COVID-19 spread occurs due to droplet transmission and touching objects used by infected patients. $65 \%$ of participants say that mouth mask, handwash, and social isolation protects from the transmission of infection. The majority of the urban population responded that the urban areas are more prone to COVID-19 infection than the rural areas. However, there was no significant difference between the opinion of urban and rural populations on the infection-prone areas, p-value $=0.687(>0.05)$. The majority of the urban population $(41 \%)$ believes that coronavirus spreads through both the routes (droplets and touching objects used by infected patients) and the rural population responded less $(24 \%)$ for both the routes of transmission, p-value $=0.016(<0.05)$, hence it was statistically significant. More of the urban population (27\%) believes that the lockdown was very useful in minimizing the coronavirus transmission when compared to the rural population $(15 \%)$, p-value $=0.004(<0.05)$, hence it was statistically significant.

Conclusion: According to the study, rural people are less aware of Covid-19 than urban people. Rural people need far more knowledge and awareness about COVID-19 for prevention and control of infection.

Key Words: Urban people, Coronavirus, Rural people, Awareness, Perception, Pandemic.

\section{INTRODUCTION}

COVID-19 is an ongoing global pandemic. ${ }^{1}$ Coronavirus are enveloped viruses with a positive-sense single-stranded RNA genome and a nucleocapsid of helical symmetry. ${ }^{2}$ It is enfolded in a shell of icosahedral protein. ${ }^{3}$ The genome size of coronaviruses ranges from about 26 to 32 kilobases, one of the largest among RNA viruses. They have characteristic club-shaped spikes that project from their surface, creating an image reminiscent of the solar corona in electron micrographs from which their name has been derived. Many countries are trying to develop vaccines against COVID-19.
The development of vaccines against COVID-19 is a major challenge that prevails all over the world. ${ }^{4}$ Coronavirus causes an infection in the nose, sinuses, or upper throat. Most coronaviruses are not dangerous. In early 2020, after a December 2019 outbreak in China, the World Health Organization identified SARS-CoV-2 as a new type of coronavirus. The outbreak quickly started spreading around the world.COVID-19is a disease caused by SARS-CoV-2 that can cause respiratory tract infection. It can affect the upper respiratory tract (sinuses, nose, and throat) or lower respiratory tract (windpipe and lungs). The SARS-CoV-2 is also found to be detectable in urine samples of affected patients. ${ }^{5}$

\section{Corresponding Author:}

Dr. Balaji Ganesh S, Scientist, White Lab - Materials Research Centre, Saveetha Dental College and Hospitals, Saveetha Institute of Medical and Technical Sciences, Saveetha University, Chennai -77, Tamilnadu, India; Email: balajiganeshs.sdc@saveetha.com

ISSN: 2231-2196 (Print)

Received: 20.07 .2020
ISSN: 0975-5241 (Online)

Revised: 22.08 .2020
Accepted: 30.09 .2020 
Infections range from mild to deadly.SARS-CoV-2 is one of seven types of coronavirus, including those causing severe diseases such as the Middle East respiratory syndrome (MERS) and severe acute respiratory syndrome (SARS). The other coronaviruses cause most of the flu or colds that affects many people but do not pose a serious threat to otherwise healthy people. ${ }^{6} 2019$ novel coronavirus (COVID-19) is an infection that can spread directly or indirectly from one person to another, causing respiratory diseases ranging from the common cold to acute respiratory syndrome. This virus was first discovered in Wuhan, China. ${ }^{7}$ COVID-19, according to the World Health Organization, is a serious health concern and has a higher risk of severe illness and is spreading rapidly throughout the world. A total of 87,137 confirmed cases were reported worldwide, until March 3rd, 2020. ${ }^{8}$ Personal hygiene and social distancing are important to prevent the entry of viruses. Conventional and herbal mouthwashes can be used to maintain oral hygiene. ${ }^{9,10,11}$ Patients with hypertension, cardiovascular disease, diabetes, and immunocompromised patients are at higher risk of developing COVID-19. Many complications of COVID-19 can result from a condition known as cytokine release syndrome or a cytokine storm. ${ }^{12,13}$ It happens when an infection causes the immune system to flood the bloodstream with cytokines called inflammatory proteins. The patients with COVID-19 should be protected from nosocomial infection in hospitals..$^{14,15}$

The coronavirus can destroy tissues and damage the vital organs. The main transmission of this virus is by droplet transmission when talking, touching items that are used by the infected person, and physical contact. Washing hands with a disinfectant, sanitizing the hand, maintain social distancing, and wearing PPE (personal protective equipment) like mask and gloves are precautionary measures to prevent the spread of the virus. ${ }^{16}$ It can also be controlled by boosting the level of immunity and drugs like remdesivir and hydroxychloroquine are usually given to the affected people. ${ }^{17}$ Patients with COVID-19 have increased cytokine levels which may lead to liver damage. ${ }^{18}$ Anti-inflammatory drugs can be used to subside the associated fever and pain symptoms. Since people need the adequate knowledge to protect them from infection, the government and various health agencies must take steps to prevent and control COVID-19. ${ }^{19}$ Most importantly, they need to focus on teaching mass people and using all the facilities to control the disease spread. ${ }^{20}$ Therefore, this survey study aims and was designed to assess the knowledge and awareness among rural and urban citizens about COVID-19.

\section{MATERIALS AND METHODS}

A descriptive cross-sectional survey of 100 participants was conducted. Before the scheduling of the study, the official permission was obtained from the Institutional ethical committee (ethical approval number-SRB/SDC/
BDS/002/01). For the data collection, the electronic distribution of the prepared questionnaire was done by using Google Forms(https://docs.google.com/forms/d/e/1FAIpQLSc_eBOuWTLSTTJmLke6izxLfk9OTr4eNKvE83ZIPhztdxvUcg/ viewform?edit requested=true\&usp=embed facebook). 17 questions were asked in the survey. The questionnaires were typed in Google forms and shared using Whatsapp to specific communities which include both rural (Ammapettai, Kachirapalayam - Kallakurichi district) and urban (Trichy, Salem, Madurai, and Chennai cities) people of Tamilnadu, India. The electronic method is the best way to approach a large number of community peoples for data collection and as well as it is suitable in the present condition where people have to avoid gathering for the prevention of COVID-19. The participants were asked to fill the Google forms and the data was collected in an excel sheet. The questions were simple and understandable, with multiple-choice questions format.

\section{Statistical analysis}

The gathered data was analyzed using SPSS software and a Chi-square test was done. The results were considered to be statistically significant if the p-value is $<0.05$.

\section{QUESTIONNAIRE}

\footnotetext{
1. Area of Locality? (Urban /Rural)

2. Do you think urban people are more prone to coronavirus than the rural people? ( Yes / No/Maybe /Don't know )

3. What is the full form of COVID - 19? (Coronavirus disorder 2029 / Coronavirus disease 2019 / Coronavirus infection disease 2019)

4. Do you think both urban and rural people are aware of COVID - 19? ( Yes / No / Maybe / Don't know )

5. Coronavirus was first identified at which place? (Wuhan, Hubei / Beijing / shanghai )

6. What do you think are the main causes of coronavirus transmission? (Droplet transmission / Touching object used by infected patients / Both A and B / All transmission )

7. What is the incubation period for coronavirus? ( 14 days / 14 weeks / 12 days / 7 days )

8. Symptoms of COVID - 19? ( Cough, difficult in breathing / Fever / Body pain / Both A and B)

9. What steps would prevent coronavirus transmission? ( Social isolation / Hand wash / Face mask / All of the above )

10. Is there any treatment for coronavirus? ( Yes / No / Don't Know )

11. Do you think immune boosting will help in the reduction of infection? ( Yes / No / Maybe / Don't Know )

12. What is the natural remedy can you take for control of coronavirus? (Vitamin $C$ Supplement / Vegetables / Both A and B / Mineral Supplement )

13. Do you think the lockdown period is necessary to control virus transmission? (It is very useful / Moderately useful / Not useful to people / People are most affected )

14. Do You think protective face masks and gloves can reduce the risk of virus transmission? ( Yes / No / Maybe / Don't Know )

15. Is there any treatment for Coronavirus? ( Yes / No / Don't know )

16. Do you think immune boosting will help in the reduction of infection? (Yes / No / Maybe / Don't Know

17. Who is the most affected in COVID - 19? (Children / Old age people / Adult / Both A and $B$ / All the above)
}

\section{RESULTS}

The questionnaires were distributed to 100 participants belonging to both rural and urban parts of Tamil Nadu, India. $48 \%$ of the study population was males and $52 \%$ were females (Figure 1). $53 \%$ of the study population was urban and $47 \%$ belong to rural areas (Figure 2). $66 \%$ of participants accept that the urban population is more prone to the spread of coronavirus infection than the urban population (Figure 3). 
$60 \%$ of participants agree that both urban and rural people are aware of coronavirus (Figure 4 ). $70 \%$ of participants say that coronavirus infection was first identified in Wuhan city (Figure 5). 65\% of participants say that COVID-19 spread occurs due to droplet transmission and touching objects used by infected patients (Figure 6). 66\% of participants say that the incubation period for coronavirus is 14 days (Figure 7). $59 \%$ of participants say that fever and difficulty in breathing, coughing are common symptoms of coronavirus (Figure 8 ). $65 \%$ of participants say that mouth mask, handwash, and social isolation protects from the transmission of infection (Figure 9).

$32 \%$ of participants say that there are treatments for coronavirus disease (Figure 10). 64\% of participants say that vitamin $\mathrm{C}$ and vegetable supplements help in maintaining health and are natural remedies to control the infection (Figure 11). 53\% of participants say that PPE helps in the prevention of infection (Figure 12). $45 \%$ of participants say that both children and old age people are commonly affected with coronavirus infection (Figure 13). The majority of the urban population responded that the urban areas are more prone to COVID-19 infection than the rural areas. However, there was no significant difference between the opinion of urban and rural populations on the infection-prone areas. P-value $=0.687(>0.05)$ (Figure 14). The majority of the urban population believes that coronavirus spreads through both the routes (droplets and touching objects used by infected patients) $(41 \%)$ and the rural population responded less $(24 \%)$ for both the routes of transmission. P-value $=0.016(<0.05)$, hence, it was statistically significant (Figure 15). More of the urban population $(27 \%)$ believes that the lockdown was very useful in minimizing the coronavirus transmission when compared to the rural population $(15 \%)$. P-value $=0.004(<0.05)$, hence, it was statistically significant (Figure 16).

\section{DISCUSSION}

The world is witnessing the impact of COVID-19 in all facts of life. This survey was done to assess the perception and awareness among rural and urban citizens about COVID-19. COVID-19 had affected both urban and rural parts of India. In the current study, 53\% were urban and $47 \%$ were from rural areas. $60 \%$ agreed that both urban and rural populations are aware of coronavirus disease. From another crosssectional study; 95 percent of urban people are aware of COVID-19 mainly in the Bihar area. ${ }^{21} 55.3$ percent of them received COVID-19 related multimedia information, from television, radio, newspaper, and 21.4 percent from the internet, followed by 14.6 percent from their family or friends in the rural community. In another cross-sectional analysis which was done to assess the awareness, attitudes, and practices of coronavirus among the Saudi public, it was found that participants showed high levels of concern and had used precautionary measures to protect from the viral infection and their knowledge of coronavirus was found to be significant. $^{22}$

In the present study, $70 \%$ said correctly that coronavirus infection was first identified in Wuhan city. The vast majority of the participants also kept a positive outlook towards the COVID-19 pandemic. ${ }^{23,24}$ Another study stated that $90.8 \%$ had assumed that COVID-19 would eventually be managed effectively and 97.1 percent had confidence that China could win the fight against the virus. ${ }^{25}$ In present study, $65 \%$ prefer combination techniques such as mouth mask usage, handwash, and social isolation to protect from the transmission of infection. Social distancing at health care facilities is necessary to prevent infection from spreading. A sufficient amount of running water taps should be accessible at the entrance of all hospitals. Free provisions of soaps and sanitizers can be made available. The habits developed included people avoiding crowded areas $(96.4 \%)$ and wore masks while leaving the home $(98.0 \%){ }^{26}$ Because of limited access to the Internet and online health information resources, vulnerable populations are more likely to have poor knowledge, negative attitudes, and inappropriate preventive practices towards COVID-19 under the COVID-19 pandemic, such as older adults and rural people at the grass-root level. ${ }^{27}$

Participants in our study demonstrated a positive general attitude towards measures that can be followed to prevent the disease from transmitting. ${ }^{28}$ They believed in the value of handwashing and their personal contact was limited. While about three-quarters of participants believed it was possible to protect against infection by putting a face mask, only about $35 \%$ were willing to do so. ${ }^{29}$ In present study, $53 \%$ believe that personal protective equipment helps in the prevention of coronavirus infection. Recently, the CDC has recommended compulsory masks usage for the public, particularly in areas where significant community-based transmission exists. ${ }^{30}$

In present study, 59\% think that fever and difficulty in breathing, coughing are common symptoms of coronavirus. $64 \%$ say that vitamin $C$ and vegetable supplements are natural remedies to control viral infection. $32 \%$ believe that there is a treatment for coronavirus disease. Antiviral drugs are given in affected individuals, analgesics to control fever symptoms and supportive patient care are also being followed as vaccine development is still going on all over the world. On the other hand, if a person has respiratory symptoms or cares for another person the WHO recommends using face masks only. Masks are more effective in the control of disease because they have the ability to prevent larger expelled droplets of individuals from evaporating into smaller droplets that can travel even farther. ${ }^{31}$

In the current study, it is found that there is a significant difference in the opinion among the urban and rural population, that the majority of the urban population believes that 
coronavirus spreads through both the routes (droplets and by touching the objects used by infected patients) and the rural population responded less for both the routes of transmission. It is also found that the urban population believes that the lockdown is very useful in minimizing coronavirus disease transmission than the rural population. These comparisons were statistically significant. The challenge faced in this study was no proper education about coronavirus, language issues, and difficulties in sharing the form of Google with rural people. The limitation of the study was that there was poor understanding efficiency among rural people, lack of interest to fill the form, language problem, and poor network availability among participants. Less sample size is also a limitation. The difference of opinions was not collected from the vast population.

\section{CONCLUSION}

From the study, it is found that there is a significant difference in the opinion about coronavirus among the urban and rural population, that the majority of the urban population are more aware of coronavirus transmission and safety measures while rural people were less aware of coronavirus transmission and preventive measures. It is found that the urban population believes that the lockdown is very useful in minimizing the coronavirus transmission than the rural population. We conclude that rural people are less aware of COVID-19 than urban people. Rural people need much more information and understanding about novel COVID-19 disease which helps in the prevention and control of the disease. The findings of this study show that it is necessary to provide adequate knowledge for each individual and to put their knowledge into practice, after which our mission will be successful in preventing the spread and transmission of this novel disease from our community, country, and also globally.

\section{ACKNOWLEDGEMENT}

Authors acknowledge the immense help received from the scholars whose articles are cited and included in references to this manuscript.

\section{Sources of funding - None \\ Conflicts of interest $-\mathrm{Nil}$}

\section{REFERENCES}

1. Lamb LE, Bartolone SN, Ward E, Chancellor MB. Rapid detection of novel coronavirus/Severe Acute Respiratory Syndrome Coronavirus 2 (SARS-CoV-2) by reverse transcription-loop-mediated isothermal amplification. PLoS One2020 $12 ; 15(6): \mathrm{e} 0234682$.
2. Sirinarumitr T,John P. Kluge,Prem S. Paul. Development of molecular techniques for the detection and pathogenesis study of swine corona-and corona-like virus .1998 - lib.dr.iastate.edu 23.p vol3.

3. Parikh S, Desai M, Parikh R. The Coronavirus: What you Need to Know about the Global Pandemic. Penguin Random House India Private Limited; 2020. 224 p.

4. Wilson K, Jenner E, Roberts N. Coronavirus: A Book for Children. Nosy Crow; 2020. 28 p.

5. Zhu N, Zhang D, Wang W, Li X, Yang B, Song J, et al. A novel coronavirus from Patients with Pneumonia in China. N Engl J Med 2020;382(8):727-733.

6. Arshad S, Kilgore P, Chaudhry ZS, et al. Treatment with hydroxychloroquine, azithromycin, and combination in patients hospitalized with COVID-19. Int J Infect Dis 2020;97:396-403.

7. Meena RP. Covid - 19: Coronavirus perfect weapon or just coincidence. New Era Publication;vol 21:23

8. Mallya PD, D'Silva R. Impact of Covid-19 crisis on the global economy and other sectors worldwide. Idea Publishing; 2020. $352 \mathrm{p}$.

9. Ashwin KS, Muralidharan NP. Vancomycin-resistant enterococcus (VRE) vs Methicillin-resistant Staphylococcus Aureus (MRSA). Indian J Med Microbiol2015;33 Suppl S1:166-7.

10. Girija ASS, Smiline Girija AS, Vijayashree Priyadharsini J, Paramasivam A. Plasmid-encoded resistance to trimethoprim/ sulfamethoxazole mediated by dfrA1, dfrA5, sul1 and sul2 among Acinetobacter baumannii isolated from urine samples of patients with severe urinary tract infection.J Glob Antimicrob Resist 2019;17:145-6.

11. Selvakumar R, Np M. Comparison in benefits of herbal mouthwashes with chlorhexidine mouthwash: A review.Asian Journal of Pharmaceutical and Clinical Research2017;10:3-7.

12. Wang J, Jiang M, Chen X, Montaner LJ. Cytokine storm and leukocyte changes in mild versus severe SARS-CoV-2 infection: Review of 3939 COVID-19 patients in China and emerging pathogenesis and therapy concepts.J Leukoc Biol 2020;108(1):17-41.

13. Ruggiero A, Megna M, Annunziata MC, Abategiovanni L, Scalvenzi M, Tajani A, et al. Teledermatology for acne during COVID-19: high patients' satisfaction in spite of the emergency.J Eur Acad Dermatol Venereol 2020;10.1111/jdv.16746.

14. Shahana R.Y, MuralidharanN.P. Efficacy of Mouth Rinse in Maintaining Oral Health of Patients Attending Orthodontic Clinics. Research J. Pharm. and Tech 2016; 9(11):1991-1993.

15. Vaishali M, Geetha RV. Antibacterial activity of Orange peel oil on Streptococcus mutans and Enterococcus-An In-vitro study. Research J. Pharm. and Tech 2018;11(2):513-514.

16. M MA, Geetha RV, Thangavelu L.Evaluation of anti-inflammatory action of Laurus nobilis-an invitro study. International Journal of Research in Pharmaceutical Sciences 2019;10:12091213.

17. Girija As S, Priyadharsini J V. CLSI based antibiogram profile and the detection of MDR and XDR strains of Acinetobacter baumannii isolated from urine samples. Med J Islam Repub Iran2019;33(3):11-16.

18. Paramasivam A, Vijayashree Priyadharsini J, Raghunandhakumar S. N6-adenosine methylation (m6A): a promising new molecular target in hypertension and cardiovascular diseases. Hypertens Res 2020;43(2):153-154.

19. Girija SAS, Jayaseelan VP, Arumugam P. Prevalence of VIMand GIMproducing Acinetobacter baumannii from patients with severe urinary tract infection. Acta Microbiologica et Immunologica Hungarica 2018;16(8):539-550. 
20. Smiline A, Vijayashree JP, Paramasivam A. Molecular characterization of plasmid-encoded blaTEM, blaSHV and blaCTX$\mathrm{M}$ among extended spectrum $\beta$-lactamases [ESBLs] producing Acinetobacter baumannii. Br J Biomed Sci 2018;75(4):200-202.

21. Priyadharsini JV, Vijayashree Priyadharsini J, Smiline Girija AS, Paramasivam A. An insight into the emergence of Acinetobacter baumannii as an oro-dental pathogen and its drug resistance gene profile - An insilico approach. Heliyon 2018;4(12):e01051.

22. Priyadharsini JV, Vijayashree Priyadharsini J, Smiline Girija AS, Paramasivam A. In silico analysis of virulence genes in an emerging dental pathogen A. baumannii and related species. Archives of Oral Biology 2018;94:93-98.

23. Shahzan MS, Sohaib Shahzan M, Smiline Girija AS, Vijayashree Priyadharsini J. A computational study targeting the mutated L321F of ERG11 gene in C. albicans, associated with fluconazole resistance with bioactive compounds from Acacia nilotica.J Mycol Med 2019;29(4):303-309.

24. Mathew M. What Is Coronavirus aka Covid19? Protect Yourself \& Others! DIY Facemask, Sanitizers \& How to isolate yourself: Myths Busters \& much more to fight against Corona Virus. Maester Books;2015 vol 12;120 p.

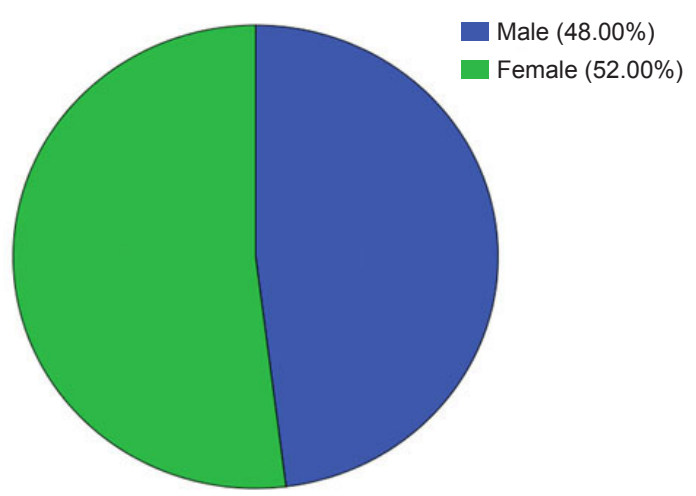

Figure 1: Pie chart showing the gender distribution of the study population. $48 \%$ (blue) of the study population were males and $52 \%$ (green) of the study population were females.

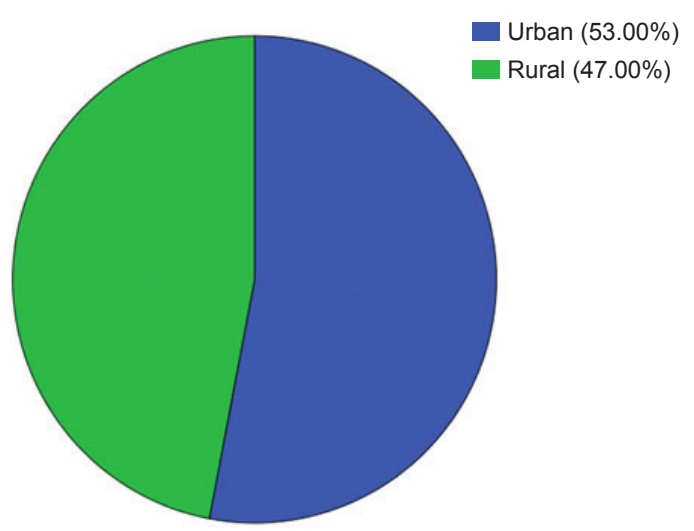

Figure 2: Pie chart showing the residence of the study population.53\%(blue) of the study population are urban and $47 \%$ (green) of the study population are rural.
25. Luqman Z, Iqbal N, Ali HM, Mustafa Z, Sikandar A, Kausar R. Disinfection of corona virus in histopathology laboratories. Clin Anat 2020;33(6):975-976.

26. Kannuchamy KS, Kesavelu D. Overview and Preventive Strategies of Coronavirus Among Healthcare Communities. Int J Cur Res Rev2020;12(15):2-6.

27. Singh G, Sharma PK, Malviya R, Awasthi R. Novel corona virus (COVID-19) disease and ophthalmic manifestations: Clinical evidences. Dermatol Ther 2020; e13814.

28. Chinese Society of Nephrology; Chinese Medical Association Professional Committee of Nephrology, Chinese Research Hospital Association. Zhonghua Nei Ke Za Zhi 2020;59(0):E014.

29. Chatterjee P, Nagi N, Agarwal A, Das B, Banerjee S, Sarkar S, Gupta N, Gangakhedkar RR. The 2019 novel coronavirus disease (COVID-19) pandemic: A review of the current evidence. Indian J Med Res 2020;151:147-159.

30. Totura AL, Bavari S. Broad-spectrum coronavirus antiviral drug discovery. Expert Opin Drug Discov 2019;14(4):397-412.

31. Esposito S, Principi N, Leung CC, Migliori GB. Universal use of face masks for success against COVID-19: evidence and implications for prevention policies. Eur Respir J2020;55(6):2001260.

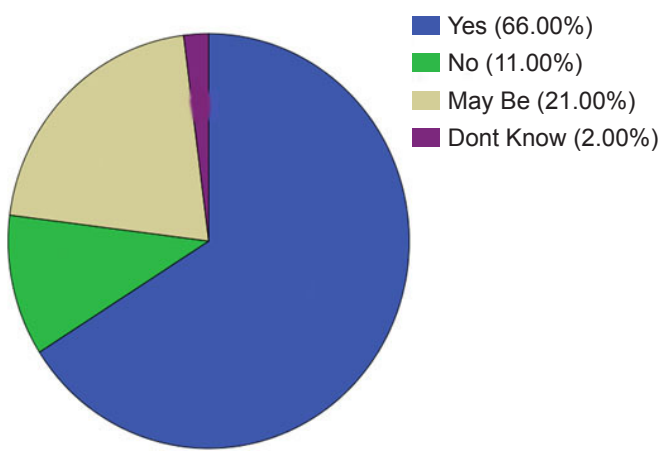

Figure 3: Pie chart depicting the opinion of the study population on the spread of corona infection which shows that $66 \%$ (blue) of participants accept that urban population more prone to spread of infection than rural population.11\%(green) participants do not accept that urban population more prone to spread of infection than rural population.21\%(brown) participants say it may be that urban population more prone to spread of infection than rural population. $2 \%$ (Purple)participants don't know whether the urban population was more prone to spread of infection than the rural population.

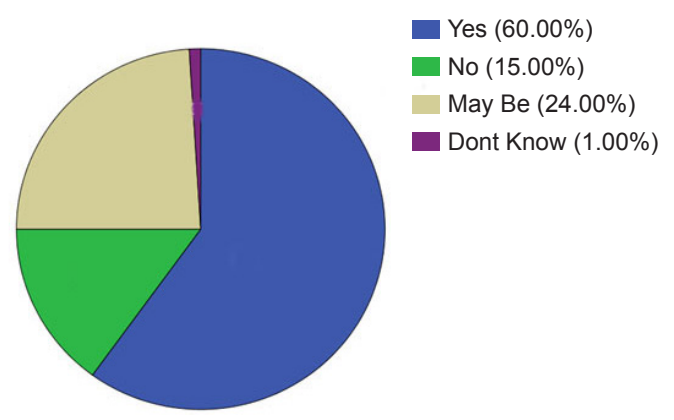

Figure 4: Pie chart depicting the opinion of the study population on awareness of COVID-19 among both urban and rural people. $60 \%$ (blue) participants agree thatboth urban and rural people are aware.15\% (green) participants do not agree.24\% (brown) participants say it may be. $1 \%$ (purple) participants don't know. 


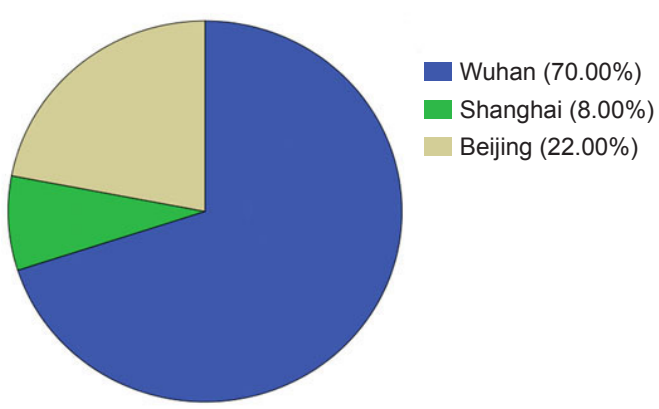

Figure 5: Pie chart depicting the opinion of the study population on awareness of identification of coronavirus. $70 \%$ (blue) of participants say that it was first identified in wuhan.8\% (green) of participants say that it was first identified in shangai.22\% (brown)of participants say that it was first identified in beijing.

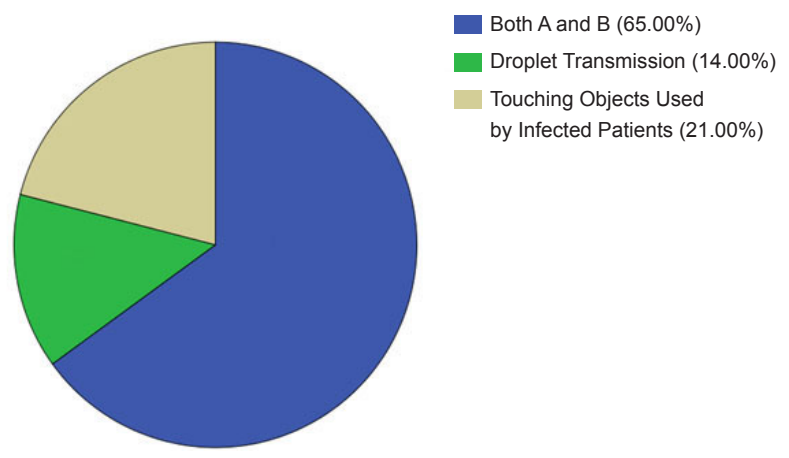

Figure 6: Pie chart depicting the opinion of the study population on the spread of coronavirus infection. 14\% (green) participants say that transmission may be due to droplet transmission. $21 \%$ (brown) of participants say that transmission may be due to objects touched by infected patients.65\% (blue) of participants say that it may be due to droplet transmission and touching objects used by infected patients.

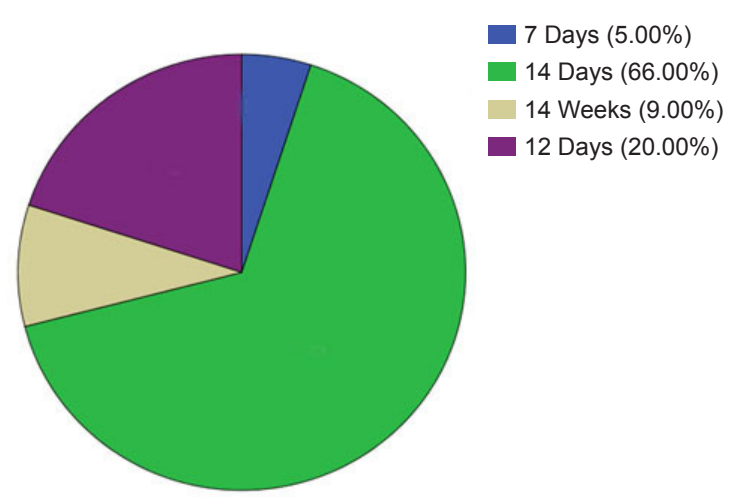

Figure 7: Pie chart depicting the opinion of the study population on awareness about the incubation period for coronavirus. $5 \%$ (blue) of participants say that the incubation period for coronavirus is 7 days. $66 \%$ (green) participants say that the incubation period for coronavirus is 14 days. 9\% (brown) of participants say that the incubation period is 14 weeks. $20 \%$ (purple) of participants say that the incubation period for coronavirus is 12 days.

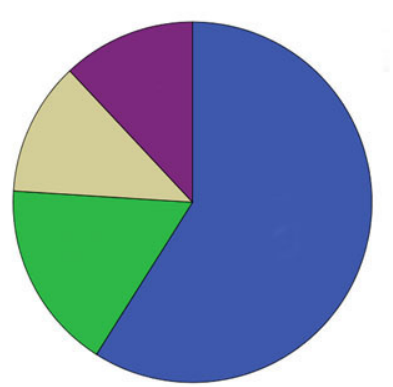

Both A and B (59.00\%)

Fever $(17.00 \%)$

Body Pain (12.00\%)

Cought Difficulty in Breathing (12.00\%)
Figure 8: Pie chart depicting the opinion of the study population on awareness about symptoms of coronavirus. $17 \%$ (green) of participants say fever was the symptom for COVID-19.12\% (brown) participants say that body pain was the symptom for COVID-19.12\% (purple) of participants say that coughing and breathing difficulty. 59\%(blue) of participants says that fever and difficulty in breathing, coughing are the symptoms for coronavirus.

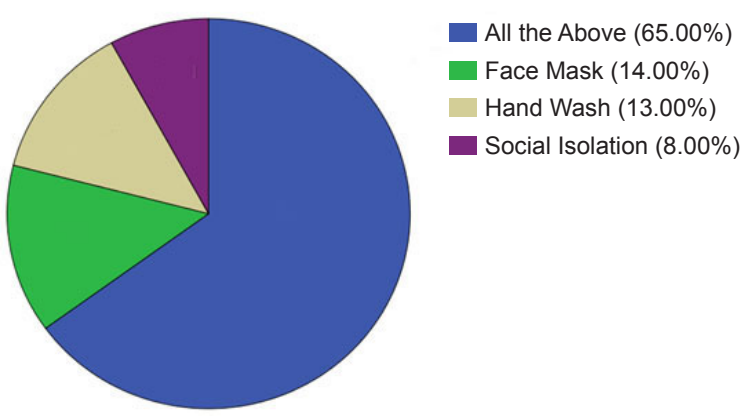

Figure 9: Pie chart depicting the opinion of the study population on control of coronavirus transmission. 14\%(green) of participants say that the face mask protects against the transmission. $13 \%$ (brown) participants say that hand wash protects against the transmission. $8 \%$ (purple) participants say that social isolation protects from infection transmission.65\% (blue) of participants say that face mask, hand wash and social isolation protects from the transmission of infection.

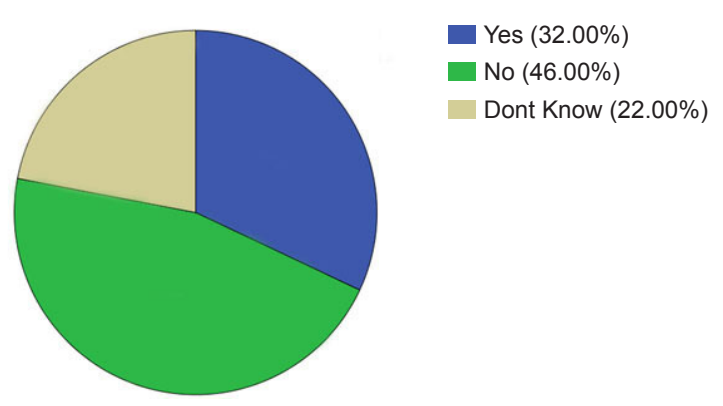

Figure 10: Pie chart depicting the opinion of the study population on awareness about treatment about coronavirus infection. $32 \%$ (blue) of participants say that there is treatment for coronavirus. while $46 \%$ (green) of participants say that there is no treatment for coronavirus, $22 \%$ (brown) of participants don't know about the treatment for coronavirus. 


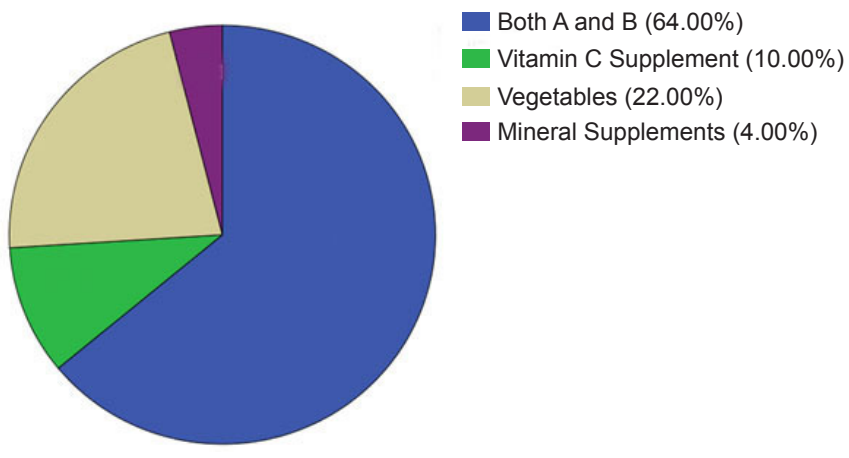

Figure 11: Pie chart depicting the opinion of the study population on awareness about natural remedy for coronavirus. $10 \%$ (green) of participants say vitamin $\mathrm{C}$ diet helps in maintaining health. $22 \%$ (brown) of participants say that vegetables helps in maintaining health. $4 \%$ (purple) participants say that mineral supplement helps in maintaining health. 64\% (blue) participants say that vitamin c supplementsand vegetables helps in maintaining health.

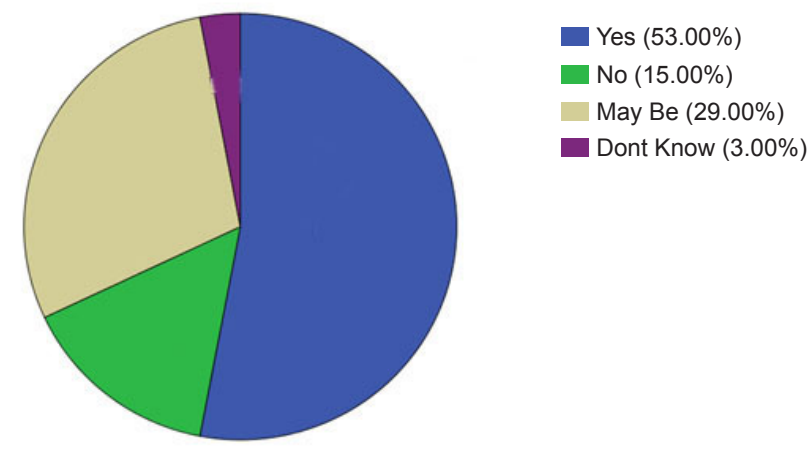

Figure12: Pie chart depicting the opinion of the study population on whether PPE helps in prevention of coronavirus. 53\% (blue) of participants say that PPE helps in the prevention of infection.15\% (green) participants say that PPE does not help in the prevention of infection.29\% (brown) participants say that it may help in the prevention of infection.3\% (purple) participants don't know whether it helps in protection against infection.

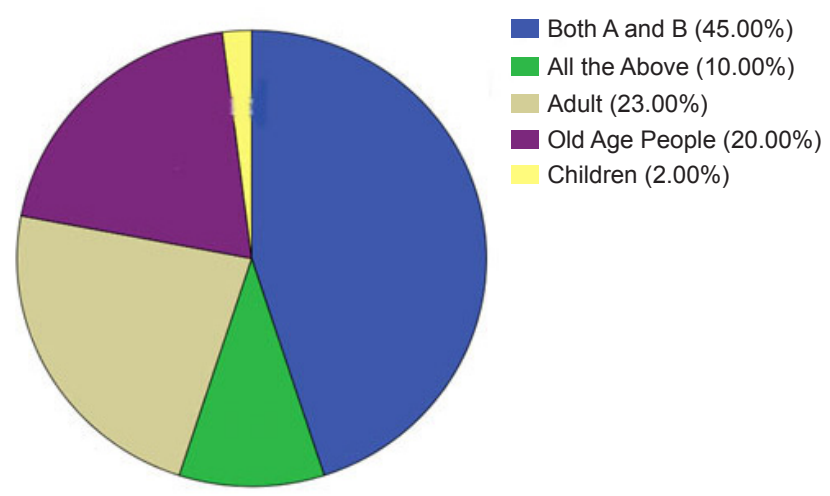

Figure 13: Pie chart depicting the opinion of the study population on awareness about coronavirus affecting age groups.
$23 \%$ (brown) of participants say that adults are commonly affected. $20 \%$ (purple) of participants say that old age people are commonly affected. $2 \%$ (yellow) participants say that children are commonly affected. $45 \%$ (blue) of participants say that both children and old age are commonly affected. 10\% (green) of participants say that all age groups are affected.

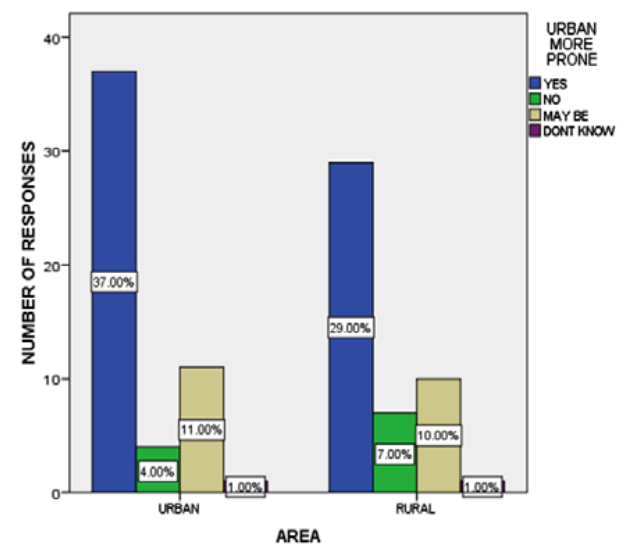

Figure 14: Bar graph depicting the association between the urban and rural population and their opinion on the locality which is more prone to coronavirus. The $\mathrm{X}$-axis represents the area of the locality of participants and Y-axis represents the number of responses. The majority of the urban population responded to the urban area being more prone than the rural population. However,there was no significant difference between the opinion of urban and rural populations on the infection-prone areas. Pearson Chi-Square test $=1.481, p$-value $=0.687(>0.05)$.

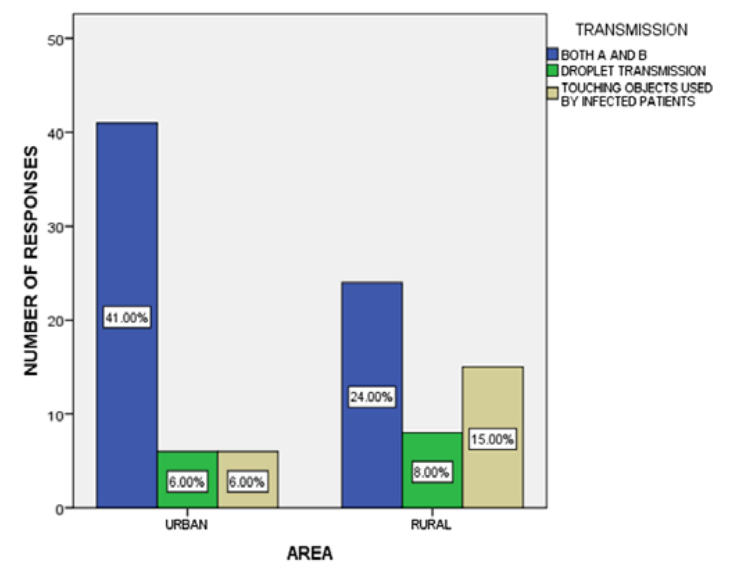

Figure 15: Bar graph depicting the association between the area of locality and awareness about coronavirus transmission. The $\mathrm{X}$-axis represents the area of locality and $\mathrm{Y}$-axis represents the number of responses. It was found that there was a significant difference in the opinion among the urban and rural population, that the majority of the urban population (41\%) believes that coronavirus spreads through both the routes(droplets and touching objects used by infected patients) and the rural population responded less $(24 \%)$ for both the routes of transmission. Pearson Chi-Square test=8.259, p-value=0.016 $(<0.05)$. 


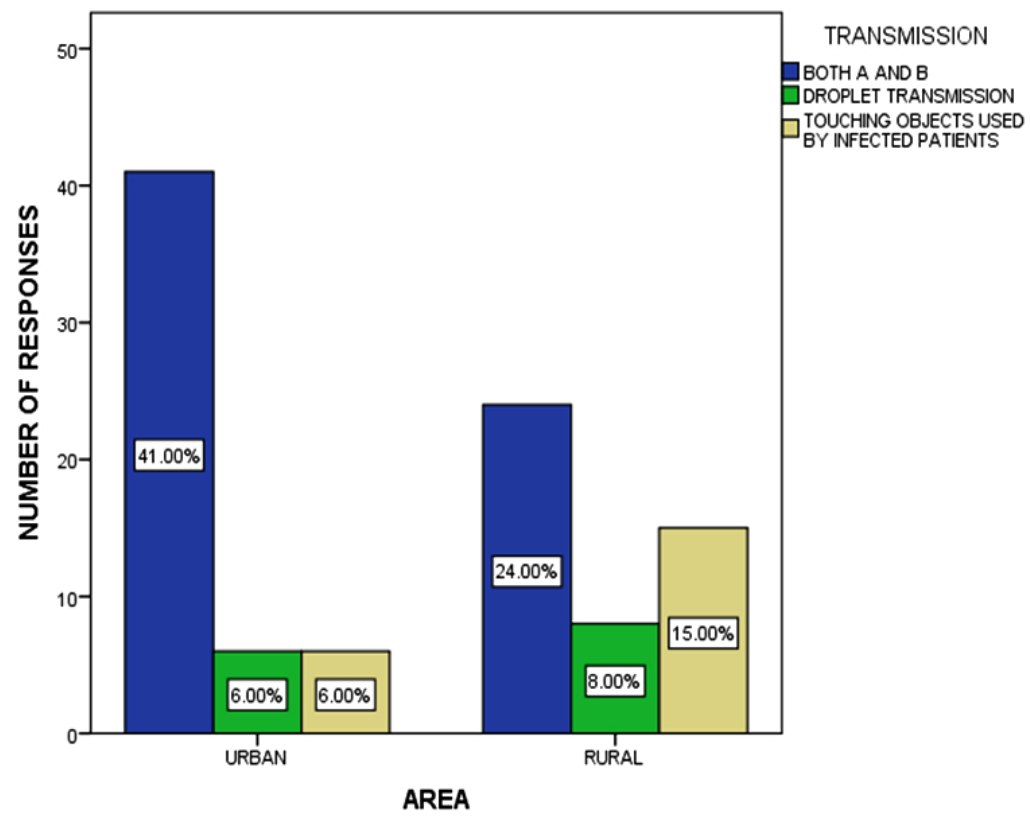

Figure 16: Bar graph depicting the association between the area of locality and usefulness of lockdown in minimizing transmission of coronavirus infection. The $\mathrm{X}$-axis represents the area of locality and $\mathrm{Y}$-axis represents the number of responses. It was found that there is a significant difference statistically, that the urban population $(27 \%)$ believes that the lockdown was very useful in minimizing the coronavirus transmission than the rural population(15\%). Pearson Chi-Square test $=13.271, \mathrm{p}-$ value $=0.004(<0.05)$. 